\title{
Epidemiology of patients with central nervous system infections, mainly neurosurgical patients: a retrospective study from 2012 to 2019 in a teaching hospital in China
}

\author{
Zheng Zhang ${ }^{1 \dagger}$, Yan Song ${ }^{2 \dagger}$, Jianbang Kang ${ }^{2}$, Surong Duan ${ }^{3}$, Qi Li ${ }^{1}$, Fuqiang Feng ${ }^{4^{*}}$ and Jinju Duan ${ }^{2^{*}}$
}

\begin{abstract}
Background: Central nervous system (CNS) infections are relatively rare but are associated with high mortality worldwide. Empirical antimicrobial therapy is crucial for the survival of patients with CNS infections, and should be based on the knowledge of the pathogen distribution and antibiotic sensitivities. The aim of this study was to investigate the features of pathogens in patients with CNS infections in North China and evaluate the risk factors for mortality and multi-drug-resistant (MDR) bacterial infections.

Methods: A retrospective study was conducted with patients with positive cerebrospinal fluid (CSF) cultures in a teaching hospital from January 2012 to December 2019. The following data were collected: demographic characteristics, laboratory data, causative organisms and antimicrobial sensitivity results. Data were analyzed with SPSS 16.0. Univariate analysis and binary logistic regression analyses were performed to identify the risk factors for mortality and MDR bacterial infections.

Results: A total of 72 patients were diagnosed with CNS infections, and 86 isolates were identified. The proportions of Gram-positive bacteria, Gram-negative bacteria and fungi were 59.3, 30.2 and 10.5\%, respectively. The predominant Gram-positive bacteria was Coagulase-negative Staphylococci. Acinetobacter baumannii, Escherichia coli and Klebsiella spp. were the predominant Gram-negative bacteria. Compared to 2012-2015 years, the proportion of Gram-negative bacteria increased markedly during 2016-2019 years. Coagulase-negative Staphylococci, Streptococcus pneumoniae and Enterococcus faecium had $100 \%$ sensitivity to vancomycin, teicoplanin and linezolid. Acinetobacter baumannii and Klebsiella pneumoniae were 100\% sensitive to tigecycline. Escherichia coli had 100\% sensitivity to amikacin, meropenem and imipenem. The overall mortality rate in the 72 patients was 30.6\%. In multivariate analysis, age > 50 years, pulmonary infections and CSF glucose level $<$ the normal value were associated with poor outcomes. CSF adenosine deaminase level > the normal value and the presence of external ventricular drainage/lumbar cistern drainage were associated with MDR bacterial infections.
\end{abstract}

\footnotetext{
*Correspondence: doctorfengfuqiang@163.com; duanjinju@163.com

${ }^{\dagger}$ Zheng Zhang and Yan Song contributed equally to this work

2 Department of Pharmacy, Second Hospital of Shanxi Medical University,

No 382, Wuyi Road, Xinghualing District, Taiyuan, Shanxi, People's

Republic of China

${ }^{4}$ Department of Neurosurgery, Second Hospital of Shanxi Medical

University, No 382, Wuyi Road, Xinghualing District, Taiyuan, Shanxi,

People's Republic of China

Full list of author information is available at the end of the article
}

(C) The Author(s) 2021. Open Access This article is licensed under a Creative Commons Attribution 4.0 International License, which permits use, sharing, adaptation, distribution and reproduction in any medium or format, as long as you give appropriate credit to the original author(s) and the source, provide a link to the Creative Commons licence, and indicate if changes were made. The images or other third party material in this article are included in the article's Creative Commons licence, unless indicated otherwise in a credit line to the material. If material is not included in the article's Creative Commons licence and your intended use is not permitted by statutory regulation or exceeds the permitted use, you will need to obtain permission directly from the copyright holder. To view a copy of this licence, visit http://creativecommons.org/licenses/by/4.0/. The Creative Commons Public Domain Dedication waiver (http://creativeco mmons.org/publicdomain/zero/1.0/) applies to the data made available in this article, unless otherwise stated in a credit line to the data. 
Conclusions: The mortality rate due to CNS infections reached $30.6 \%$ in our study. The proportion of Gram-negative bacteria has increased markedly in recent years. We should give particular attention to patients with risk factors for mortality and MDR bacterial infections mentioned above.

Keywords: Central nervous system infections, North China, Pathogens distribution, Antibiotic susceptibility, Risk factors

\section{Background}

Central nervous system (CNS) infections, including meningitis, encephalitis, and brain abscesses, can occur as complications following neurosurgical operations or they can occur spontaneously $[1,2]$. CNS infections cause significant mortality worldwide, resulting in a poor prognosis with a prolonged hospital stay and increased costs [1-5]. More than 100 pathogens have been reported to cause CNS infections, including bacteria, viruses, fungi and parasites [5]. The most common bacterial pathogens are Neisseria meningitidis, Streptococcus pneumoniae, Coagulase-negative Staphylococci (CoNS), Staphylococcus aureus and Acinetobacter baumannii [6, 7]. Cerebrospinal fluid (CSF) culture is commonly used to determine the pathogens causing CNS infections. However, the final results of culture, including antimicrobial susceptibility testing, cannot be obtained within $48 \mathrm{~h}[8,9]$. Gram staining and agglutination tests can only preliminarily distinguish among Gram-positive, Gram-negative and fungi. Thus, empirical antimicrobial therapy is essential while the pathogen identification and antimicrobial susceptibility test results are still pending, and should be based on the knowledge of the prevalence of various bacterial organisms and their antibiotic sensitivity. However, antibiotic therapy for CNS infections has been limited due to the inefficiency of drug transport across the blood-brain barrier (BBB) and the emergence of multidrug-resistant (MDR) bacteria [6, 10-12]. In addition, the prevalence of pathogens causing bacterial infections varies based on time, geographical distribution and underlying medical conditions [13]. China has a vast territory, and the pathogen distribution differs across the country. Thus, the availability of local bacterial prevalence and antibiotic sensitivity data could help improve the empirical administration of antimicrobial therapy.

Furthermore, understanding the risk factors for mortality and MDR bacterial infections would assist clinicians in implementing interventions in a timely manner to improve patient outcomes. There were numerous studies reported the potential risk factors associated with the incidence of CNS infections in recent decades [14-16], however, data on the risk factors associated with mortality and MDR bacterial infections are limited. Herein, we present epidemiological research on CNS infections at a tertiary teaching hospital in North China and assess the crucial risk factors affecting patient outcomes and the development of MDR bacterial infections.

\section{Methods \\ Data collection}

This retrospective study was carried out at the Second Hospital of Shanxi Medical University, a 2700-bed tertiary teaching hospital in Shanxi, China. This study registered in an eight-year period (from January 2012 to December 2019). The data from patients with positive CSF cultures reported by the microbiological laboratory were obtained from the electronic medical records. For each patient, three main sets of records were collected. The first set of records was related to the general demographic characteristics, including age, sex, department, comorbidities, hospital length of stay, surgery and placement of invasive devices. The second set of records was related to the pathogen isolates and their antimicrobial susceptibility results. Duplicate isolates from a patient within 7 days were excluded. The third set of records included the following laboratory test results: the glucose level, protein level, chlorine level, adenosine deaminase (ADA) level, leukocyte count and erythrocyte count in the CSF; the white blood cell count, neutrophil count and percentage of neutrophils in the blood; the levels of liver function indicators, including alanine aminotransferase (ALT), aspartate aminotransferase (AST), total bilirubin (TBIL), alkaline phosphatase (ALP), total protein and albumin; and the levels of renal function indicators, including creatinine and urea nitrogen. We included the results of laboratory tests performed close to the time at which the CSF samples were collected.

\section{Microbiology and antimicrobial susceptibility testing}

After the CSF samples were received, centrifugation and gram stain were performed to suggest the bacterial species initially. Blood agar plates, chocolate agar plates and MacConkey agar plates were used for bacterial culture, and plates are incubated in carbon dioxide at $35{ }^{\circ} \mathrm{C}$. Isolates were identified by the Vitek 2 automated system (Biomerieux, France). Antimicrobial susceptibility of bacteria was tested by Kirby-Bauer disk diffusion method and interpreted according to the latest standards of Clinical and Laboratory Standards Institute (CLSI) Guidelines. 


\section{Definitions}

CNS infections was diagnosed according to the definitions of Centers for Disease Control and Prevention (CDC) as follows [17]: (1) isolation of pathogens from CSF; (2) patient was considered at least one of the following signs with no other recognized cause: fever $\left(>38^{\circ} \mathrm{C}\right)$, headache, stiff neck, meningeal signs, cranial nerve signs, changing level of consciousness, or confusion; (3) increased white cells, elevated protein, and/or decreased glucose in CSF. MDR is defined as non-susceptibility to three or more classes of antibiotics, and extensively drugresistant (XDR) is defined as non-susceptibility to at least one agent in all but two or fewer antimicrobial categories $[18,19]$. The study population was divided into two groups respectively according to whether the patients survived and whether the patients detected with MDR bacteria in the CSF.

\section{Statistical analysis}

Data were analyzed with SPSS software version 16.0. The statistical results for continuous data were recorded as mean \pm SD or median (IQR) values according to the statistical distribution, and categorical parameters as number (\%). To compare the difference between two groups of patients (survivors/non-survivors or MDR-positive/ MDR-negative), categorical variables were analyzed by a Chi-square test or Fisher's extract test, as required. Independent samples t-test was used for continuous variables with normal distribution, and continuous variables with non-normal distribution were compared by using the independent samples Mann-Whitney U test. Two-tailed tests were used to determine statistical significance and a $P$ value $<0.05$ was considered statistically significant. Factors with a $P$ value $<0.1$ in univariate Chi-square test or Fisher's extract test were included in a binary logistic regression model to identify the independent risk factors. Laboratory data were converted to dichotomous variables by selecting a cut-off point based on the maximum or minimum referenced values.

\section{Results}

\section{Study population}

From January 2012 to December 2019, we obtained 111 positive CSF culture samples from 98 patients; 22 patients were excluded because they did not meet the diagnostic criteria for a CNS infections. In addition, 4 patients were removed because they were diagnosed with viral meningitis by the attending clinicians. Finally, 72 patients were diagnosed with CNS infections caused by bacteria or fungi, and 86 isolates were identified. Table 1 shows the characteristics of the study population.
Table 1 Clinical characteristics of the study population $(\mathrm{N}=72)$

\begin{tabular}{ll}
\hline Characteristics & Value \\
\hline Age (years) & $49.7 \pm 18.3$ \\
Age $>50$ & $41(56.9)$ \\
Sex & \\
Male & $54(75)$ \\
Female & $18(25)$ \\
Department & \\
Neurosurgery & $52(72.2)$ \\
Neurology & $15(20.8)$ \\
Other & \\
Days in hospital & $5(6.9)$ \\
Comorbidity & $29.5(19.3-46.0)$ \\
Pulmonary infection & \\
Hypertension & $35(48.6)$ \\
Hydrocephalus & $29(40.3)$ \\
Intracranial hemorrhage & $26(36.1)$ \\
Diabetes & $19(26.4)$ \\
Glucocorticoid therapy $>3$ days & $10(13.9)$ \\
Surgery & $29(40.3)$ \\
Yes & \\
No & $50(69.4)$ \\
Invasive device & $22(30.6)$ \\
External ventricular drainage/Lumbar cistern drainage & $41(56.9)$ \\
Catheter & $57(79.2)$ \\
\hline Rheumatogy (n & \\
\hline &
\end{tabular}

${ }^{a}$ Rheumatology $(n=1)$, Cardiothoracic surgery $(n=1)$, Oncology $(n=1)$, Hematology $(n=1)$, Intensive Care Unit $(n=1)$

\section{Microbiology}

The distribution of the 86 causative agents is shown in Table 2. Among all the microorganisms detected, the proportions of Gram-positive bacteria, Gram-negative bacteria and fungi were 59.3, 30.2 and $10.5 \%$, respectively. The predominant Gram-positive isolate was CoNS (43.0\%), followed by Enterococcus faecium (5.8\%) and Streptococcus pneumoniae (5.8\%). Acinetobacter baumannii (7.0\%), Escherichia coli (7.0\%) and Klebsiella spp. (5.8\%) were the common Gram-negative strains. In addition, 9 fungal isolates were identified, 6 of which were Cryptococcus neoformans.

The changes in pathogen distribution across different periods are shown in Fig. 1 and Table 2. We observed that Gram-positive bacteria were still the dominant pathogens, but the detection rate of Gram-negative bacteria increased markedly. The proportion of Gram-negative bacteria relative to the total number of pathogens was $15.4 \%$ in the period from 2012 to 2015 and $42.6 \%$ in the period from 2016 to 2019. Moreover, fungi were also found more frequently in the later period. In particular, Streptococcus pneumoniae and Klebsiella spp. were identified only in the period from 2016 to 2019. Furthermore, 
Table 2 Pathogens isolated from patients with CNS infections

\begin{tabular}{|c|c|c|c|}
\hline Pathogens & n (\%) & $\begin{array}{l}2012- \\
2015 \text { years } \\
\text { n (\%) }\end{array}$ & $\begin{array}{l}2016- \\
2019 \text { years } \\
\text { n (\%) }\end{array}$ \\
\hline Total & 86 & 39 & 47 \\
\hline Gram-positive bacteria & $51(59.3)$ & $30(76.9)$ & $21(44.7)$ \\
\hline $\begin{array}{l}\text { Coagulase-negative Staphylo- } \\
\text { cocci }^{{ }^{a}}\end{array}$ & $37(43.0)$ & $24(61.5)$ & $13(27.7)$ \\
\hline Enterococcus faecium & $5(5.8)$ & $4(10.3)$ & $1(2.1)$ \\
\hline Streptococcus pneumoniae & $5(5.8)$ & & $5(10.6)$ \\
\hline Staphylococcus aureus & $2(2.3)$ & $2(5.1)$ & \\
\hline Enterococcus faecalis & $1(1.2)$ & & $1(2.1)$ \\
\hline Streptococcus anginosus & $1(1.2)$ & & $1(2.1)$ \\
\hline Gram-negative bacteria & $26(30.2)$ & $6(15.4)$ & $20(42.6)$ \\
\hline Acinetobacter baumannii & $6(7.0)$ & $1(2.6)$ & $5(10.6)$ \\
\hline Escherichia coli & $6(7.0)$ & $3(7.7)$ & $3(6.4)$ \\
\hline Klebsiella spp. ${ }^{b}$ & $5(5.8)$ & & $5(10.6)$ \\
\hline Enterobacter cloacae & $2(2.3)$ & & $2(4.3)$ \\
\hline Enterobacter aerogenes & $2(2.3)$ & & $2(4.3)$ \\
\hline Pseudomonas aeruginosa & $1(1.2)$ & & $1(2.1)$ \\
\hline Acinetobacter Iwoffii & $1(1.2)$ & & $1(2.1)$ \\
\hline Stenotrophomonas maltophilia & $1(1.2)$ & $1(2.6)$ & \\
\hline Pseudomonas oryzihabitans & $1(1.2)$ & & $1(2.1)$ \\
\hline Brucella spp. & $1(1.2)$ & $1(2.6)$ & \\
\hline Fungus & $9(10.5)$ & $3(7.7)$ & $6(12.8)$ \\
\hline Cryptococcus neoformans & $6(7.0)$ & $2(5.1)$ & $4(8.5)$ \\
\hline Aspergillus fumigatus & $1(1.2)$ & & $1(2.1)$ \\
\hline Candida parapsilosis & $1(1.2)$ & $1(2.6)$ & \\
\hline Candida tropicalis & $1(1.2)$ & & $1(2.1)$ \\
\hline
\end{tabular}

a Staphylococcus haemolyticus ( $n=11)$, Staphylococcus epidermidis $(n=4)$, Staphylococcus hominis $(n=1)$, unidentified Coagulase-negative Staphylococci $(\mathrm{n}=21)$

${ }^{\mathrm{b}}$ Klebsiella pneumoniae $(\mathrm{n}=3)$, Klebsiella oxytoca $(\mathrm{n}=2)$

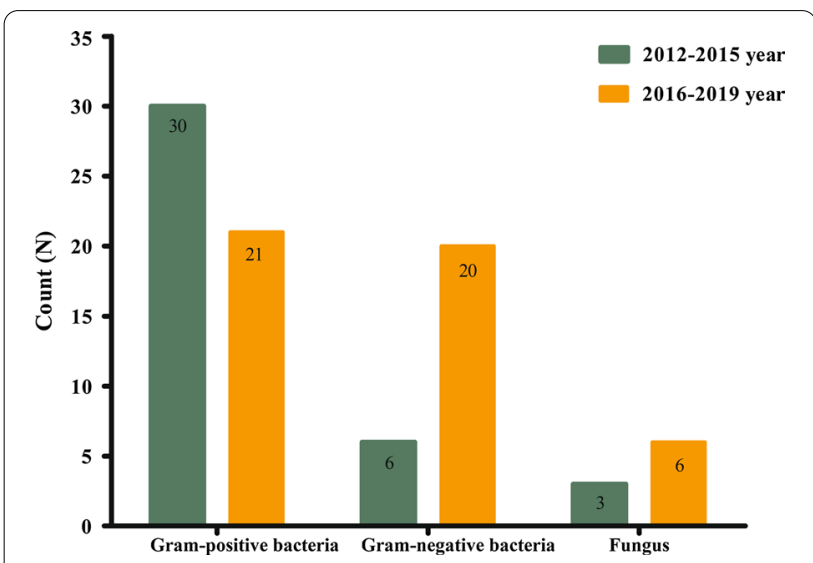

Fig. 1 The pathogens distribution in different periods we compared the pathogen distributions between the neurosurgery and non-neurosurgery groups (Table 3).

\section{Antimicrobial susceptibility testing}

The in vitro antibiotic sensitivities of the Gram-positive isolates are shown in Table 4 . CoNS had $80 \%$ sensitivity to rifampicin and $100 \%$ sensitivity to vancomycin, teicoplanin and linezolid. All isolates of Streptococcus pneumoniae were sensitive to levofloxacin, moxifloxacin vancomycin and teicoplanin. For the Enterococcus faecium, vancomycin, teicoplanin and linezolid were $100 \%$ sensitive antibiotics. Of the Gram-negative isolates, Acinetobacter baumannii were $83.3 \%$ sensitive to minocycline, and no isolates were resistant to tigecycline. Escherichia coli had $100 \%$ sensitivity to amikacin, meropenem and imipenem. For Klebsiella pneumoniae, the only antibiotic to which there was no resistance was tigecycline. The details of the antibiotic sensitivity of the Gram-negative isolates are shown in Table 5. For the fungal isolates, Cryptococcus neoformans had $100 \%$ sensitivity to 5-fluorouracil, amphotericin B, fluconazole and voriconazole.

\section{Risk factors for mortality}

The demographic and clinical characteristics of both survivors and non-survivors with CNS infections are shown in Table 6. Of the study population, 50 patients survived, and 22 patients died, yielding a mortality rate of $30.6 \%$. In univariate analysis, we found significant differences between the two groups in the following aspects $(\mathrm{P}<0.05)$ : age, pulmonary infection, hypertension, the CSF glucose level, the CSF protein level, the CSF leukocyte count, the blood percentage of neutrophils, the ALT level, the AST level, the serum albumin level, and the urea nitrogen level. In binary logistic regression analysis, we found that age $>50$ years, pulmonary infection, and a CSF glucose level $<$ the normal value were independent risk factors for mortality (Table7).

\section{Risk factors for MDR bacterial infections}

Compared with MDR bacteria-negative patients, MDR bacteria-positive patients more frequently underwent surgery, had external ventricular drainage/lumbar cistern drainage, were complicated with intracranial haemorrhage, and had elevated levels of CSF ADA, protein and leukocytes (Table 8). According to the logistic regression model, a CSF ADA level > the normal value and the presence of external ventricular drainage/lumbar cistern drainage were associated with MDR bacterial infections (Table 9). 
Table 3 Pathogens distribution of neurosurgery and non-neurosurgery groups

\begin{tabular}{|c|c|c|c|}
\hline Pathogens & n (\%) & Neurosurgery n (\%) & $\begin{array}{l}\text { Non- } \\
\text { neurosurgery }{ }^{1} \\
\mathrm{n}(\%)\end{array}$ \\
\hline Total & 86 & 64 & 22 \\
\hline Gram-positive bacteria & $51(59.3)$ & $43(67.2)$ & $8(36.4)$ \\
\hline Coagulase-negative Staphylococcia & $37(43.0)$ & $35(54.7)$ & $2(9.1)$ \\
\hline Enterococcus faecium & $5(5.8)$ & $5(7.8)$ & \\
\hline Streptococcus pneumoniae & $5(5.8)$ & $1(1.6)$ & $4(18.2)$ \\
\hline Staphylococcus aureus & $2(2.3)$ & $1(1.6)$ & $1(4.5)$ \\
\hline Enterococcus faecalis & $1(1.2)$ & & $1(4.5)$ \\
\hline Streptococcus anginosus & $1(1.2)$ & $1(1.6)$ & \\
\hline Gram-negative bacteria & $26(30.2)$ & $19(29.7)$ & $7(31.8)$ \\
\hline Acinetobacter baumannii & $6(7.0)$ & $6(9.4)$ & \\
\hline Escherichia coli & $6(7.0)$ & $4(6.3)$ & $2(9.1)$ \\
\hline Klebsiella spp. ${ }^{b}$ & $5(5.8)$ & $3(4.7)$ & $2(9.1)$ \\
\hline Enterobacter cloacae & $2(2.3)$ & $1(1.6)$ & $1(4.5)$ \\
\hline Enterobacter aerogenes & $2(2.3)$ & $2(3.1)$ & \\
\hline Pseudomonas aeruginosa & $1(1.2)$ & $1(1.6)$ & \\
\hline Acinetobacter Iwoffii & $1(1.2)$ & & $1(4.5)$ \\
\hline Stenotrophomonas maltophilia & $1(1.2)$ & $1(1.6)$ & \\
\hline Pseudomonas oryzihabitans & $1(1.2)$ & $1(1.6)$ & \\
\hline Brucella spp. & $1(1.2)$ & & $1(4.5)$ \\
\hline Fungus & $9(10.5)$ & $2(3.1)$ & $7(31.8)$ \\
\hline Cryptococcus neoformans & $6(7.0)$ & & $6(27.3)$ \\
\hline Aspergillus fumigatus & $1(1.2)$ & & $1(4.5)$ \\
\hline Candida parapsilosis & $1(1.2)$ & $1(1.6)$ & \\
\hline Candida tropicalis & $1(1.2)$ & $1(1.6)$ & \\
\hline
\end{tabular}

${ }^{a}$ Staphylococcus haemolyticus $(n=11)$, Staphylococcus epidermidis $(n=4)$, Staphylococcus hominis $(n=1)$, unidentified Coagulase-negative Staphylococci $(n=21)$

${ }^{\mathrm{b}}$ Klebsiella pneumoniae $(\mathrm{n}=3)$, Klebsiella oxytoca $(\mathrm{n}=2)$

${ }^{1}$ Department of Neurology, Rheumatology, Cardiothoracic surgery, Oncology, Hematology, Intensive Care Unit

\section{Discussion}

The microbiological examination of CSF specimens serves as an important basis for the diagnosis and treatment of CNS infections. We retrospectively collected the data from patients with positive CSF culture results and analyzed the pathogen distribution, antibiotic sensitivity and risk factors for mortality. Furthermore, risk factors for MDR bacterial infections were assessed. In our study, 111 positive CSF culture specimens were identified. According to the results of retrospective analysis, 26 samples from 26 patients were considered to be false positives in our 8-year study. The occurrence of false positive culture result could be due to a lack of standardization of the available assays and uncertainty about sample collection and processing. In addition, the CSF samples could have been contaminated by normal microbial species on the skin, such as CoNS. Wong et al. and Chang et al. reported proportions of false-positive CSF culture results of $58.7 \%(71 / 121)$ and $11.1 \%(12 / 108)$, respectively [12, 20]. Thus, it is important to exclude false-positive results.
Of the 86 isolates detected in patients with confirmed CNS infections, the proportions of Gram-positive bacteria, Gram-negative bacteria and fungi in our study were $59.3,30.2$ and $10.5 \%$, respectively. This was approximately the same as the results of previous studies $[12,21]$. In our study, the top seven pathogens were CoNS (43.0\%), Acinetobacter baumannii (7.0\%), Escherichia coli (7.0\%), Cryptococcus neoformans (7.0\%), Enterococcus faecium (5.8\%), Streptococcus pneumoniae (5.8\%) and Klebsiella spp. (5.8\%). These results did not differ significantly from those of the multi-center study of positive CSF isolates from the China Antimicrobial Surveillance Network (CHINET, www.chinets.com), as they showed that CoNS was the most common isolate ( $>44.97 \%)$, followed by Acinetobacter baumannii (12.43\%), Klebsiella pneumoniae (8.23\%), Enterococcus faecium (3.95\%), Escherichia coli (3.92\%), Staphylococcus aureus (2.61\%) and Enterococcus faecalis $(2.42 \%)$ in 2019 . Whereas, Cryptococcus neoformans accounted for a higher percentage in our study. Our data indicated that the age range of the 
Table 4 Antimicrobial susceptibility of Gram-positive isolates

\begin{tabular}{|c|c|c|c|c|c|c|c|c|c|c|c|c|}
\hline & \multicolumn{4}{|c|}{ CoNS $(\mathrm{N}=37)$} & \multicolumn{4}{|c|}{ Streptococcus pneumoniae $(\mathrm{N}=5)$} & \multicolumn{4}{|c|}{ Enterococcus faecium $(\mathrm{N}=5)$} \\
\hline & $\mathbf{n}$ & $\mathrm{S}$ & I & $\mathbf{R}$ & $\mathbf{n}$ & $S$ & I & $\mathbf{R}$ & $\mathbf{n}$ & $S$ & I & $\mathbf{R}$ \\
\hline Penicillin & 37 & $1(2.7 \%)$ & & $36(97.3 \%)$ & 5 & $2(40 \%)$ & & $3(60 \%)$ & 5 & & & $5(100 \%)$ \\
\hline Oxacillin & 37 & $7(18.9 \%)$ & & $30(81.1 \%)$ & - & & & & - & & & \\
\hline Ampicillin & - & & & & - & & & & 5 & & & $5(100 \%)$ \\
\hline Amoxicillin-Clavulanate & 34 & $7(20.6 \%)$ & & $27(79.4 \%)$ & - & & & & - & & & \\
\hline Cefazolin & 28 & $4(14.3 \%)$ & & $24(85.7 \%)$ & - & & & & - & & & \\
\hline Ciprofloxacin & 16 & $5(31.3 \%)$ & & $11(68.8 \%)$ & - & & & & 5 & & & $5(100 \%)$ \\
\hline Levofloxacin & 20 & $8(40 \%)$ & $5(25 \%)$ & $7(35 \%)$ & 5 & $5(100 \%)$ & & & - & & & \\
\hline Moxifloxacin & 6 & $3(50 \%)$ & $1(16.7 \%)$ & $2(33.3 \%)$ & 5 & $5(100 \%)$ & & & - & & & \\
\hline Tetracycline & 36 & 27 (75\%) & & $9(25 \%)$ & 5 & & $1(20 \%)$ & $4(80 \%)$ & 5 & $3(60 \%)$ & & $2(40 \%)$ \\
\hline Erythrocin & 37 & $4(10.8 \%)$ & & $33(89.2 \%)$ & 5 & & $1(20 \%)$ & $4(80 \%)$ & - & & & \\
\hline Clindamycin & 37 & $14(37.8 \%)$ & $7(18.9 \%)$ & $16(43.2 \%)$ & 3 & & & $3(100 \%)$ & - & & & \\
\hline Chloramphenicol & - & & & & 5 & $4(80 \%)$ & & $1(20 \%)$ & 5 & $3(60 \%)$ & $2(40 \%)$ & \\
\hline Rifampicin & 35 & $28(80 \%)$ & & $7(20 \%)$ & 2 & $2(100 \%)$ & & & 5 & $1(20 \%)$ & & $4(80 \%)$ \\
\hline Compound Sulfamethoxazole & 37 & $9(24.3 \%)$ & & $28(75.7 \%)$ & 5 & $2(40 \%)$ & $1(20 \%)$ & $2(40 \%)$ & - & & & \\
\hline Gentamicin & 37 & $21(56.8 \%)$ & $1(2.7 \%)$ & $15(40.5 \%)$ & - & & & & 5 & $1(20 \%)$ & & $4(80 \%)$ \\
\hline Vancomycin & 37 & $37(100 \%)$ & & & 5 & $5(100 \%)$ & & & 5 & $5(100 \%)$ & & \\
\hline Teicoplanin & 37 & $37(100 \%)$ & & & 5 & $5(100 \%)$ & & & 5 & $5(100 \%)$ & & \\
\hline Linezolid & 37 & $37(100 \%)$ & & & 2 & $2(100 \%)$ & & & 5 & $5(100 \%)$ & & \\
\hline
\end{tabular}

$S$ sensitive, / intermediary, $R$ resistant, (-) not done

6 patients with Cryptococcus neoformans CNS infections was between 43 and 68 years, of whom 1 patient had tumour, two patients had hypoproteinemia and four patients had electrolyte disturbances.

However, according to the published data, the most common pathogens causing CNS infections are Neisseria meningitidis, Streptococcus pneumoniae and Haemophilus influenzae [22, 23]. Our results were significantly different. There are several possible reasons: (1) Neisseria meningitidis, Streptococcus pneumoniae and Haemophilus influenzae are fastidious bacteria, and they are difficult to grow under normal culture conditions. (2) Neisseria meningitidis, Streptococcus pneumoniae and Haemophilus influenzae are sensitive to most antimicrobial agents, and initial antimicrobial therapy may have contributed to the low positive culture rate. (3) In our study, 52 out of 72 patients were from neurosurgery and the majority of patients (56.9\%) had undergone external ventricular drainage/lumbar cistern drainage. For patients with neurosurgery-related CNS infections, most studies have identified Staphylococcus spp. as the most common pathogens, particularly CoNS [12, 14], which was consistent with our results.

Therefore, we divided the patients into two groups by department. The results showed that in the neurosurgery group, the main pathogens were CoNS (54.7\%), Acinetobacter baumannii (9.4\%) and Enterococcus faecium (7.8\%), while in the non-neurosurgery group,
Cryptococcus neoformans (27.3\%) and Streptococcus pneumoniae $(18.2 \%)$ were the predominant pathogens. Recognizing the differences in pathogen distribution in different departments could be inform the choice of corresponding empirical treatment.

With respect to the pathogen distribution in different periods, we found that the proportion of Gram-negative bacteria increased markedly from $15.4 \%$ in the period from 2012 to 2015 to $43.5 \%$ in the period from 2016 to 2019, particularly, Acinetobacter baumannii from $2.6 \%$ to $10.6 \%$ and Klebsiella spp. from 0 to $10.6 \%$. In recent decades, CNS infections caused by Gram-negative bacteria have attracted more attention from clinicians, especially MDR/XDR Acinetobacter baumannii and Enterobacteriaceae [24-28]. Of the Gram-positive bacteria, CoNS was still the predominant pathogen, although the percentage decreased dramatically from 61.5 to $27.7 \%$. In addition, compared to the period from 2012 to 2015, the number of species of isolated strains increased during the period from 2016 to 2019, and Streptococcus pneumoniae and Klebsiella spp. were identified only in the later period. The cultivation of Streptococcus pneumoniae is challenging because autolysis results in decreased viability [28]. Advances in automated continuously monitored CSF culture systems could process positive bottles before autolysis occurs. Therefore, the increase in isolated species could be associated with the improvements in laboratory and microbiological inspection techniques. 


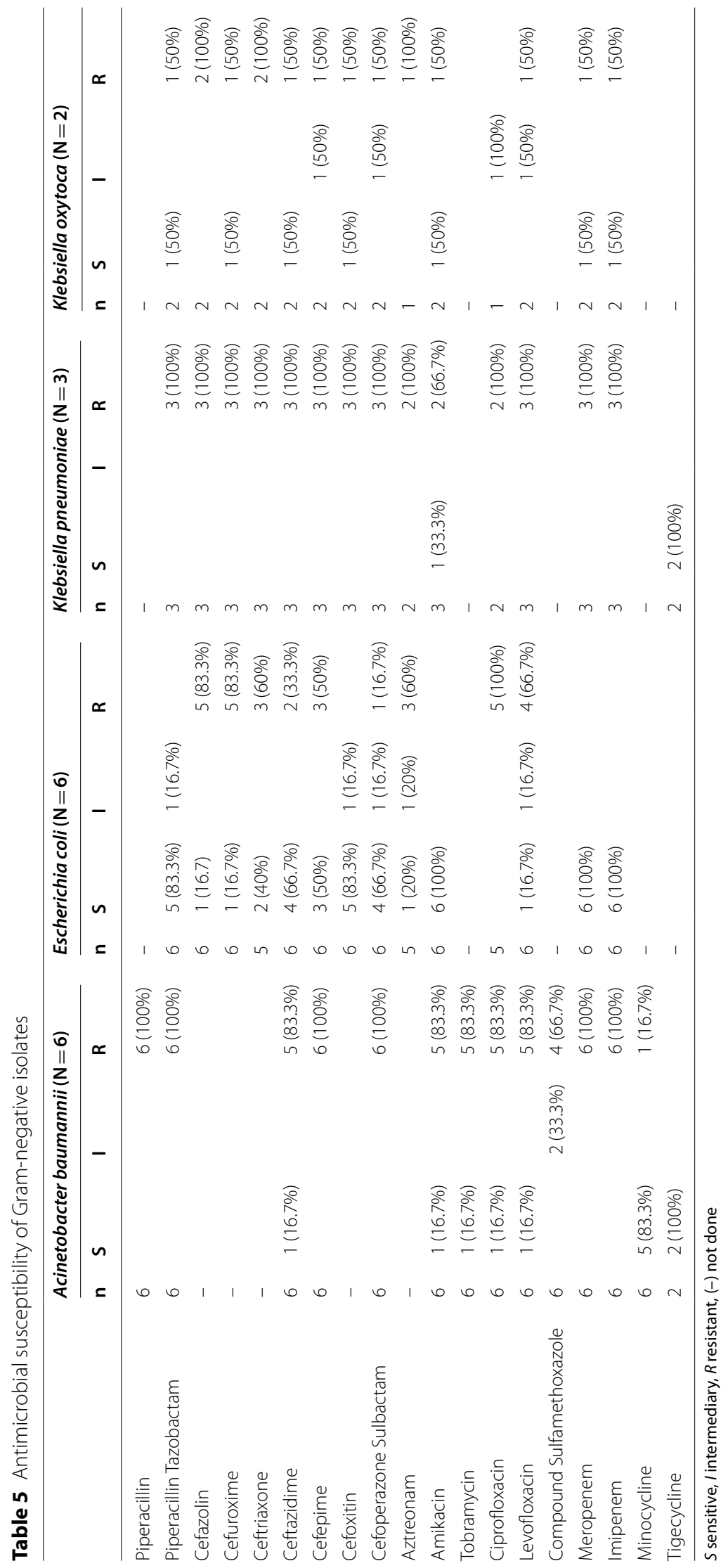


Table 6 Comparison of demographic and clinical characteristics between survivors and non-survivors

\begin{tabular}{|c|c|c|c|c|}
\hline Characteristics & Total $(\mathrm{N}=72)$ & Survivors $(\mathrm{N}=50)$ & Non-Survivors $(\mathrm{N}=22)$ & $\mathbf{P}$ \\
\hline Age (years) & $49.7 \pm 18.3$ & $45.5 \pm 17.8$ & $59.0 \pm 16.3$ & 0.003 \\
\hline Age $>50$ & 41 & 23 & 18 & 0.005 \\
\hline \multicolumn{5}{|l|}{ Sex } \\
\hline Male & $54(75.0)$ & $36(72.0)$ & $18(81.8)$ & \multirow[t]{2}{*}{0.375} \\
\hline Female & $18(25.0)$ & $14(28.0)$ & $4(18.2)$ & \\
\hline Days in hospital & $29.5(19.3-46.0)$ & $29.0(20.7-45.0)$ & $33.5(18.0-51.0)$ & 0.633 \\
\hline \multicolumn{5}{|l|}{ Comorbidity } \\
\hline Pulmonary infection & 35 (48.6) & $15(30.0)$ & $20(90.9)$ & $<0.001$ \\
\hline Hypertension & $29(40.3)$ & $16(32.0)$ & $13(59.1)$ & 0.031 \\
\hline Hydrocephalus & $26(36.1)$ & $16(32.0)$ & $10(45.5)$ & 0.274 \\
\hline Intracranial hemorrhage & $19(26.4)$ & $13(26.0)$ & $6(27.3)$ & 0.91 \\
\hline Diabetes & $10(13.9)$ & $5(10.0)$ & $5(22.7)$ & 0.285 \\
\hline MDR bacteria infection & $49(68.1)$ & $31(62.0)$ & $18(81.8)$ & 0.097 \\
\hline Glucocorticoid therapy $>3$ days & $29(40.3)$ & $18(36.0)$ & $11(50.0)$ & 0.265 \\
\hline \multicolumn{5}{|l|}{ Surgery } \\
\hline Yes & $50(69.4)$ & $33(66.0)$ & $17(77.3)$ & \multirow[t]{2}{*}{0.339} \\
\hline No & $22(30.6)$ & $17(34.0)$ & $5(22.7)$ & \\
\hline \multicolumn{5}{|l|}{ Invasive device } \\
\hline $\begin{array}{l}\text { External ventricular drainage/Lumbar } \\
\text { cistern drainage }\end{array}$ & $41(56.9)$ & $25(50.0)$ & $16(72.7)$ & 0.073 \\
\hline Catheter & $57(79.2)$ & $37(74.0)$ & $20(90.9)$ & 0.189 \\
\hline \multicolumn{5}{|l|}{ CSF data } \\
\hline ADA level (U/L) & $15.50(4.33-30.95)$ & $14.00(3.30-28.25)$ & $19.55(5.48-37.48)$ & 0.131 \\
\hline Glucose level (mmol/L) & $1.71(0.21-2.98)$ & $2.29(1.35-3.46)$ & $0.35(0.05-0.91)$ & $<0.001$ \\
\hline Chlorine level (mmol/L) & $116.68 \pm 9.27$ & $117.16 \pm 8.67$ & $115.58 \pm 10.63$ & 0.508 \\
\hline Protein level (g/L) & $2.37(1.12-4.01)$ & $1.67(0.84-2.78)$ & $4.00(2.98-4.54)$ & $<0.001$ \\
\hline Leukocyte count $\left(\times 10^{6} / \mathrm{L}\right)$ & $340.0(52.5-2400.0)$ & $188.0(27.5-927.5)$ & $1125.0(102.5-4185.0)$ & 0.029 \\
\hline Erythrocyte count $\left(\times 10^{6} / \mathrm{L}\right)$ & $395.0(10.0-4787.5)$ & $395.0(7.5-1987.5)$ & $810.0(65.0-10,675.0)$ & 0.306 \\
\hline \multicolumn{5}{|l|}{ Blood data } \\
\hline White blood cell level $\left(\times 10^{9} / \mathrm{L}\right)$ & $13.13 \pm 6.11$ & $12.42 \pm 5.64$ & $14.75 \pm 6.94$ & 0.138 \\
\hline Neutrophils level $\left(\times 10^{9} / \mathrm{L}\right)$ & $10.55(6.94-13.48)$ & $10.13(6.58-12.68)$ & $12.56(8.68-14.85)$ & 0.071 \\
\hline Percentage of neutrophils & $85.21(78.86-88.05)$ & 84.35 (76.36-87.49) & $87.14(83.40-88.88)$ & 0.039 \\
\hline \multicolumn{5}{|l|}{ Liver function } \\
\hline $\operatorname{ALT}(U / L)$ & $22.80(14.63-57.93)$ & $20.80(14.08-46.35)$ & $36.60(19.40-125.15)$ & 0.018 \\
\hline AST (U/L) & $22.60(17.03-39.45)$ & $20.05(15.53-38.03)$ & $31.80(19.25-66.28)$ & 0.014 \\
\hline TBIL (mmol/L) & $14.65(10.13-18.60)$ & $13.90(9.40-18.30)$ & $15.05(12.88-21.48)$ & 0.167 \\
\hline$A L P(U / L)$ & $89.5(72.0-127.0)$ & $82.0(70.5-118.0)$ & $104.0(77.8-170.5)$ & 0.069 \\
\hline Total protein (g/L) & $63.22 \pm 8.06$ & $64.30 \pm 8.04$ & $60.76 \pm 7.73$ & 0.086 \\
\hline Albumin (g/L) & $35.93 \pm 6.62$ & $37.75 \pm 5.51$ & $31.79 \pm 7.15$ & $<0.001$ \\
\hline \multicolumn{5}{|l|}{ Renal function } \\
\hline Urea nitrogen (mmol/L) & $5.05(3.58-6.95)$ & $4.72(2.98-6.58)$ & $6.13(4.63-8.60)$ & 0.02 \\
\hline Creatinine (mmol/L) & $61.53 \pm 25.25$ & $61.27 \pm 27.27$ & $62.14 \pm 20.51$ & 0.893 \\
\hline
\end{tabular}

According to the results of antibiotic sensitivity testing in this study, $81.1 \%(30 / 37)$ of the CoNS isolates were methicillin-resistant strains. This proportion was higher than those reported in some studies, such as $75 \%$ and approximately $55 \%-75 \%[12,29]$. Additionally, CoNS had $80 \%$ sensitivity to rifampicin. In China, rifampicin, which is usually used against tuberculosis bacilli, is rarely used to treat infections caused by other bacteria, except for severe MDR bacterial infections. Staphylococcus spp. had $100 \%$ sensitivity to vancomycin, teicoplanin and linezolid in the present study. Sixty percent (3/5) of the Streptococcus pneumoniae strains were resistant to penicillin in 
Table 7 Risk factors for mortality

\begin{tabular}{lccr}
\hline Factor & OR & 95\% Cl & \multicolumn{1}{c}{ P } \\
\hline Age $>50$ years & 4.73 & $0.86-25.94$ & 0.074 \\
$\begin{array}{l}\text { Pulmonary infection } \\
\text { CSF glucose level }<\text { the normal } \\
\text { value }\end{array}$ & 20.41 & $3.11-134.07$ & 0.002 \\
\hline
\end{tabular}

OR odds ratio, $\mathrm{Cl}$ confidence interval, the normal value of CSF glucose level was $2.2-3.8 \mathrm{mmol} / \mathrm{L}$

our study, while 5 Streptococcus pneumoniae strains were completely resistant to penicillin in an Ethiopian study [30]. The identified high level of resistance to penicillin, which is mainly used as a standard regimen for the empiric treatment of CNS infections caused by Streptococcus pneumoniae, could create difficulties with regard to clinical treatment. Moreover, several studies have reported the emergence of vancomycin-resistant Enterococcus faecium [31-33], yet, no vancomycin-resistant strains were identified in the present study.
83.3\% (5/6) of the isolates of Acinetobacter baumannii were XDR bacteria in our study, while an Indian study reported that $20.8 \%$ of Acinetobacter isolates were XDR strains [34]. An obvious difference was the sensitivity to cefoperazone-sulbactam, approximately $74 \%$ of the Acinetobacter isolates were found to be sensitive to cefoperazone-sulbactam in India [34], yet the 6 Acinetobacter baumannii isolates were all resistant in our study. The data from two Chinese hospitals indicated that

Table 9 Risk factors for MDR bacterial infections

\begin{tabular}{llll}
\hline Factor & OR & $\mathbf{9 5 \% ~ C l}$ & $\mathbf{P}$ \\
\hline CSF ADA level > thenormal value & 5.63 & $1.61-19.72$ & 0.007 \\
$\begin{array}{l}\text { External ventricular drainage/ } \\
\text { Lumbar cistern drainage }\end{array}$ & 6.02 & $1.85-19.64$ & 0.003 \\
\hline
\end{tabular}

The normal value of CSF ADA level was 0-5 U/L

Table 8 Differences between MDR-positive and MDR-negative patients

\begin{tabular}{|c|c|c|c|c|}
\hline Characteristics & Total $(\mathrm{N}=72)$ & MDR-positive $(\mathrm{N}=49)$ & MDR-negative $(\mathrm{N}=23)$ & $\mathbf{P}$ \\
\hline Age (years) & $49.7 \pm 18.3$ & $50.8 \pm 19.1$ & $47.3 \pm 16.6$ & 0.459 \\
\hline \multicolumn{5}{|l|}{ Sex } \\
\hline Male & $54(75.0)$ & $35(71.4)$ & $19(82.6)$ & \multirow[t]{2}{*}{0.307} \\
\hline Female & $18(25.0)$ & $14(28.6)$ & $4(17.4)$ & \\
\hline Days from hospitalization to detection of bacteria & $9.5(3.0-17.8)$ & $10.0(4.5-17.5)$ & $5.0(1.0-18.0)$ & 0.258 \\
\hline \multicolumn{5}{|l|}{ Comorbidity } \\
\hline Pulmonary infection & $35(48.6)$ & $27(55.1)$ & $8(34.8)$ & 0.108 \\
\hline Hypertension & $29(40.3)$ & $23(46.9)$ & $6(26.1)$ & 0.093 \\
\hline Hydrocephalus & $26(36.1)$ & $20(40.8)$ & $6(26.1)$ & 0.225 \\
\hline Intracranial hemorrhage & $19(26.4)$ & $18(36.7)$ & $1(4.3)$ & 0.004 \\
\hline Diabetes & $10(13.9)$ & $8(16.3)$ & $2(8.7)$ & 0.612 \\
\hline \multicolumn{5}{|l|}{ Surgery } \\
\hline Yes & $50(69.4)$ & $39(79.6)$ & $11(47.8)$ & \multirow[t]{2}{*}{0.006} \\
\hline No & $22(30.6)$ & $10(20.4)$ & $12(52.2)$ & \\
\hline \multicolumn{5}{|l|}{ Invasive device } \\
\hline External ventricular drainage/Lumbar cistern drainage & $41(56.9)$ & $34(69.4)$ & $7(30.4)$ & 0.002 \\
\hline Catheter & $57(79.2)$ & $42(85.7)$ & $15(65.2)$ & 0.092 \\
\hline \multicolumn{5}{|l|}{ CSF data } \\
\hline ADA level (U/L) & $15.50(4.33-30.95)$ & $23.00(5.95-36.35)$ & $6.00(1.50-17.10)$ & 0.001 \\
\hline Glucose level (mmol/L) & $1.71(0.21-2.98)$ & $1.65(0.17-2.66)$ & $2.11(0.23-3.40)$ & 0.476 \\
\hline Chlorine level (mmol/L) & $116.68 \pm 9.27$ & $116.03 \pm 9.5$ & $118.04 \pm 8.88$ & 0.395 \\
\hline Protein level (g/L) & $2.37(1.12-4.01)$ & $3.06(1.65-4.11)$ & $1.06(0.67-2.57)$ & $<0.001$ \\
\hline Leukocyte count ( $\left.\times 10^{6} / \mathrm{L}\right)$ & $340.0(52.5-2400.0)$ & $480.0(120.0-2400.0)$ & $60.0(8.0-2420.0)$ & 0.033 \\
\hline Erythrocyte count $\left(\times 10^{6} / \mathrm{L}\right)$ & $395.0(10.0-4787.5)$ & $800.0(70.0-6675.0)$ & $25.0(0.0-1560.0)$ & 0.052 \\
\hline \multicolumn{5}{|l|}{ Blood data } \\
\hline White blood cell level $\left(\times 10^{9} / L\right)$ & $13.13 \pm 6.11$ & $13.3 \pm 5.9$ & $12.9 \pm 6.7$ & 0.798 \\
\hline Neutrophils level (× 109/L) & $10.55(6.94-13.48)$ & $11.20(7.84-13.14)$ & $9.48(6.21-13.59)$ & 0.48 \\
\hline Percentage of neutrophils & $85.21(78.86-88.05)$ & $85.50(80.01-87.82)$ & $83.5(75.9-90.3)$ & 0.503 \\
\hline
\end{tabular}


Acinetobacter baumannii had low sensitivity to cefoperazone-sulbactam (5.3\% and 16.3\%) [21, 26]. This might indicate that regional and medical conditions have substantial effects on antibiotic resistance. However, high rates of resistance to carbapenems were found in our study and other reports [21, 26, 34].

In the meantime, meningitis caused by Enterobacteriaceae, particularly carbapenem-resistant Enterobacteriaceae (CRE), remains a therapeutic challenge worldwide [27]. Among the Enterobacteriaceae strains in our study, carbapenem-resistant species appeared only in Klebsiella spp. Four of five Klebsiella isolates showed resistance to carbapenems, of which three Klebsiella pneumoniae strains were all resistant to carbapenems. In our previous study, the mechanisms of resistance in carbapenem-resistant Klebsiella pneumoniae strains isolated from the CSF of patients in our hospital involved New Delhi metallo- $\beta$-lactamase and Klebsiella pneumoniae carbapenemases. KL47 and KL22KL37 were the serotypes and ST11 was the prevalent sequence type [35]. With regard to the Escherichia coli, our findings showed that they had low sensitivity to cephalosporins, but they had $100 \%$ sensitivity to carbapenem antibiotics and were highly sensitive to enzyme inhibitor compound preparations. These findings might demonstrate that the major mechanism of resistance in Escherichia coli in our hospital is the production of extended-spectrum beta-lactamases (ESBLs) rather than carbapenemases.

Antimicrobial agents have been used successfully to treat infectious diseases for a long time. Unfortunately, the misuse and overuse of antibiotics has led to increased antibiotic resistance [36]. Antimicrobial therapy for CNS infections has been complicated by the emergence of MDR strains. With regard to Gram-negative bacteria, tigecycline and polymyxin/colistin are usually used for the treatment of MDR/XDR Acinetobacter baumannii and Klebsiella pneumoniae infections [27, 37]. Nevertheless, due to the existence of the BBB, CNS infections does not show any improvement when treated with them by intravenous administration. Hence, the intrathecal (ITH) or intraventricular (IVT) administration of these two agents has been performed in recent years [38-41]. However, ITH and IVT antibiotic therapy has not been standardized [2]. Moreover, there are no guidelines for the ITH/IVT administration of tigecycline. We need to further explore the ITH/IVT administration of antimicrobial therapy to improve the outcomes in patients with CNS infections.

A previous study showed that mortality due to bacterial meningitis ranges from 10 to $20 \%$ in high-resource settings and can be as high as $50 \%$ in lower-resource settings [8]. This study showed that the mortality rate due to CNS infections was $30.6 \%$ in our hospital. Therefore, it is important to determine the risk factors affecting the outcomes of patients with CNS infections, particularly in developing countries. Previous studies predicted survival following CNS infections and suggested that age $>40$ years, the presence of external ventricular drainage, low CSF glucose levels, high CSF protein levels, a CSF leukocyte count $>200$ cells $/ \mathrm{mm}^{3}$, ICU admission, and the presence of comorbidities were risk factors for mortality $[34,42,43]$. In our analysis, age $>50$ years, complicated with pulmonary infection and a CSF glucose level $<$ the normal value were independent risk factors for mortality. Older patients are usually characterized by markedly altered organ and physiological functions that often require tailored treatment, such as individual drug administration and extracorporeal therapies. Thus, a comprehensive assessment of patient status is needed before treatment, especially for older patients. Complicated with a pulmonary infection might increase the difficulty of antibiotic therapy and lead to a poor prognosis. Low glucose level in the CSF is a crucial diagnostic criterion for CNS infections. Our findings showed that the CSF glucose level is not only an important diagnostic indicator, but also associated with a poor prognosis.

Lewin et al. reported that immunosuppressive therapy (glucocorticoids) may reduce meningeal inflammation but can also further diminish the distribution of antimicrobials throughout the CNS [10]. This could result in failure to achieve effective antimicrobial concentrations in the CNS. Twenty-nine patients were administered glucocorticoids in our study, and we analyzed the relationship between glucocorticoid therapy and patient outcomes in univariate analysis. Although the results indicated that there was not a significant association in our study, compared to the survivors, the non-survivors were more likely to have received glucocorticoid therapy (50\% vs $36 \%)$. Moreover, we found that the proportion of male (75\%) was significantly higher than that of female (25\%). The possible explanation could be that men are more likely to smoke or suffer craniocerebral trauma. Smoking is a risk factor for cerebrovascular disease [44]. Thus, men may account for a higher proportion of patients undergoing neurosurgery, and they are also more likely to develop CNS infections. However, we did not find a significant association between sex and patient outcome.

Exploring the risk factors for mortality and MDR bacterial infections could help clinicians initiate interventions in a timely manner to improve patient outcomes. Based on our results, with regard to the management of patients with CNS infection, there are several points should be considered. First, the initial empirical treatment should be based on the distributions of pathogens in different departments, and appropriate 
antibiotics that can be effectively transported across the BBB should be selected. Second, clinicians should give particular attention to patients with the independent risk factors for mortality and MDR bacterial infections mentioned above. For patients with pulmonary infections, the antibiotics selected should be useful for the treatment of both the pulmonary and CNS infections, and combination therapy should be strongly considered. Patients with external ventricular drainage/ lumbar cistern drainage are more likely to be infected by MDR bacteria, so it is crucial to keep the drainage tube unobstructed, provide adequate nursing care, and remove the drainage tube as early as possible. Meanwhile, nosocomial infection control measures should be strengthened, including the isolation of patients, disinfection of the environment, practice of appropriate hand hygiene by the medical staff and disinfection of the medical instruments. In addition, when CSF gram staining shows the presence of Gram-positive bacteria, vancomycin could be used as an empirical treatment in patients who are at risk for infection with MDR bacteria. However, there are substantial challenges regarding the selection of empirical antibiotic treatments for MDR Gram-negative bacteria. If necessary, the ITH/ IVT administration of colistin and aminoglycoside antibiotics could be considered.

The limitations of our study must be acknowledged. This was a single-center study with a small sample size, which may restrict the applicability of the findings to patients with CNS infections in other regions. Additionally, due to the limitations of retrospective studies, the drug sensitivity results for some antibiotics could not be obtained.

\section{Conclusion}

It is important to know the pathogen distribution and risk factors for adverse outcomes in patients with CNS infections. Although Gram-positive bacteria are still the primary pathogens causing CNS infections, the proportion of Gram-negative organisms has increased dramatically in recent years. The detected Gram-positive bacteria were still $100 \%$ sensitive to vancomycin in our study. But, MDR/XDR Acinetobacter baumannii and Klebsiella pneumoniae accounted for high proportions. Furthermore, age $>50$ years, complicated with pulmonary infections and a CSF glucose level $<$ the normal value were independent risk factors for mortality. A CSF ADA level $>$ the normal value and the presence of external ventricular drainage/lumbar cistern drainage were associated with MDR bacterial infections. More advanced research should be conducted on this topic.

\section{Abbreviations}

ADA: Adenosine deaminase; ALP: Alkaline phosphatase; ALT: Alanine aminotransferase; AST: Aspartate aminotransferase; BBB: Blood-brain barrier; CDC: Centers for Disease Control and Prevention; CLSI: Clinical and Laboratory Standards Institute; CNS: Central nervous system; CoNS: Coagulase-negative Staphylococci; CRE: Carbapenem-resistant Enterobacteriaceae; CSF: Cerebrospinal fluid; ESBLs: Extended-spectrum beta-lactamases; ITH: Intrathecal; IVT: Intraventricular; MDR: Multi-drug-resistant; TBIL: Total bilirubin; XDR: Extensively drug-resistant.

\section{Acknowledgements \\ We thank Second Hospital of Shanxi Medical University, for supporting this research.}

\section{Authors' contributions}

JD and FF designed the study. JK and SD collected the data. ZZ, YS, and $\mathrm{QL}$ analyzed and interpreted the data. ZZ and YS wrote the manuscript. All authors read and approved the final manuscript.

\section{Funding}

This study was supported by the Shanxi Province Natural Science Foundation (Grant number 201901D111390). The funders had no role in the design of the study and collection, analysis, and interpretation of data and in writing the manuscript.

\section{Availability of data and materials}

The data set supporting the conclusions in this article is available from the corresponding author on reasonable request.

\section{Declarations}

\section{Ethics approval and consent to participate}

This study was approved by the Ethics Committee of Second Hospital of Shanxi Medical University (Reference Number 2019YX-278). The Ethics Committee granted permission to access the raw data and they also approved the waiver of informed consent to participate in this study due to its retrospective design. All patient data were anonymous prior to the analysis.

\section{Consent for publication}

Not applicable.

\section{Competing interests}

The authors declare that they have no competing interests.

\section{Author details}

${ }^{1}$ Department of Pharmacy, School of Pharmacy, Shanxi Medical University, Taiyuan, Shanxi, People's Republic of China. ${ }^{2}$ Department of Pharmacy, Second Hospital of Shanxi Medical University, No 382, Wuyi Road, Xinghualing District, Taiyuan, Shanxi, People's Republic of China. ${ }^{3}$ Department of Information Management, Second Hospital of Shanxi Medical University, No 382, Wuyi Road, Xinghualing District, Taiyuan, Shanxi, People's Republic of China. ${ }^{4}$ Department of Neurosurgery, Second Hospital of Shanxi Medical University, No 382, Wuyi Road, Xinghualing District, Taiyuan, Shanxi, People's Republic of China.

Received: 3 March 2021 Accepted: 9 August 2021

Published online: 17 August 2021

References

1. Giovane R, Lavender P. Central nervous system infections. Prim Care. 2018:45(3):505-18.

2. Mrowczynski OD, Langan ST, Rizk EB. Intra-cerebrospinal fluid antibiotics to treat central nervous system infections: a review and update. Clin Neurol Neurosurg. 2018;170:140-58.

3. Yang $W, W u X, L i Z$, Yuan $Q, W u G, Y u$ J, et al. Trends of intra-cranial bacterial infection in patients requiring emergency neurosurgery. Surg Infect (Larchmt). 2020;21(8):677-83.

4. Singhi S, Angurana SK. Principles of management of central nervous system infections. Indian J Pediatr. 2019;86(1):52-9. 
5. Thanh TT, Casals-Pascual C, Ny NTH, Ngoc NM, Geskus R, Nhu LNT, et al. Value of lipocalin 2 as a potential biomarker for bacterial meningitis. Clin Microbiol Infect. 2020;27(5):724-30.

6. Nau R, Blei C, Eiffert H. Intrathecal antibacterial and antifungal therapies. Clin Microbiol Rev. 2020;33(3).

7. Baumgartner W, LoscherW. Re-emergence of neuroinfectiology. Acta Neuropathol. 2016:131(2):155-8.

8. Poplin V, Boulware D, Bahr N. Methods for rapid diagnosis of meningitis etiology in adults. Biomark Med. 2020;14(6):459-79.

9. Hasbun R, Rosenthal N, Balada-Llasat JM, Chung J, Duff S, Bozzette S, et al. Epidemiology of meningitis and encephalitis in the United States, 2011-2014. Clin Infect Dis. 2017;65(3):359-63.

10. Lewin JJ 3rd, Cook AM, Gonzales C, Merola D, Neyens R, Peppard WJ, et al. Current practices of intraventricular antibiotic therapy in the treatment of meningitis and ventriculitis: results from a multicenter retrospective cohort study. Neurocrit Care. 2019:30(3):609-16.

11. Zimmer AJ, BurkeVE, Bloch KC. Central nervous system infections. Microbiol Spectrum. 2016;4(3)

12. Chang JB, Wu H, Wang H, Ma BT, Wang RZ, Wei JJ. Prevalence and antibiotic resistance of bacteria isolated from the cerebrospinal fluid of neurosurgical patients at Peking Union Medical College Hospital. Antimicrob Resist Infect Control. 2018;7:41.

13. Chang C, Ye J, Yang C, Huang P, Chiang P, Lee M. Influence of third-generation cephalosporin resistance on adult in-hospital mortality from post-neurosurgical bacterial meningitis. Microbiol Immunol Infect. 2010;43(4):301-9.

14. Wang LY, Cao XH, Shi LK, Ma ZZ, Wang Y, Liu Y. Risk factors for intracranial infection after craniotomy: a case-control study. Brain Behav. 2020;10(7):e01658.

15. Zhan R, Zhu Y, Shen Y, Shen J, Tong Y, Yu H, et al. Post-operative central nervous system infections after cranial surgery in China: incidence, causative agents, and risk factors in 1,470 patients. Eur J Clin Microbiol Infect Dis. 2014;33(5):861-6.

16. Bodilsen J, Dalager-Pedersen M, van de Beek D, Brouwer MC, Nielsen H. Risk factors for brain abscess: a nationwide, population-based, nested casecontrol study. Clin Infect Dis. 2020;71(4):1040-6.

17. Horan TC, Andrus M, Dudeck MA. CDC/NHSN surveillance definition of health care-associated infection and criteria for specific types of infections in the acute care setting. Am J Infect Control. 2008;36(5):309-32.

18. Magiorakos AP, Srinivasan A, Carey RB, Carmeli Y, Falagas ME, Giske CG, et al. Multidrug-resistant, extensively drug-resistant and pandrug-resistant bacteria: an international expert proposal for interim standard definitions for acquired resistance. Clin Microbiol Infect. 2012:18(3):268-81.

19. Diamantino-Miranda J, Aguiar SI, Carrico JA, Melo-Cristino J, Ramirez M. Clonal and serotype dynamics of serogroup 6 isolates causing invasive pneumococcal disease in Portugal: 1999-2012. PLoS ONE. 2017;12(2):e0170354.

20. Wong PH, Maranich AM, Muench DF. Isolation of bacterial cerebrospinal fluid culture contaminants at a major military medical center. Diagn Microbiol Infect Dis. 2013;77(4):357-61.

21. Tian $L$, Zhang Z, Sun ZY. Pathogen analysis of central nervous system infec tions in a chinese teaching hospital from 2012-2018: a laboratory-based retrospective study. Curr Med Sci. 2019;39(3):449-54.

22. Brouwer MC, Tunkel AR, van de Beek D. Epidemiology, diagnosis, and antimicrobial treatment of acute bacterial meningitis. Clin Microbiol Rev. 2010;23(3):467-92

23. Dando SJ, Mackay-Sim A, Norton R, Currie BJ, St John JA, Ekberg JA, et al. Pathogens penetrating the central nervous system: infection pathways and the cellular and molecular mechanisms of invasion. Clin Microbiol Rev. 2014;27(4):691-726

24. Pandey S, Li L, Deng XY, Cui DM, Gao L. Outcome following the treatment of ventriculitis caused by multi/extensive drug resistance gram negative bacilli; Acinetobacter baumannii and Klebsiella pneumonia. Front Neurol. 2018;9:1174.

25. Rodriguez Guardado A, Blanco A, Asensi V, Perez F, Rial JC, Pintado V, et al. Multidrug-resistant Acinetobacter meningitis in neurosurgical patients with intraventricular catheters: assessment of different treatments. J Antimicrob Chemother. 2008;61(4):908-13.

26. Liang W, Yuan-Run Z, Min Y. Clinical presentations and outcomes of postoperative central nervous system infection caused by multi-drug-resistant/ extensively drug-resistant Acinetobacter baumannii: a retrospective study. Surg Infect (Larchmt). 2019;20(6):460-4.
27. Guanghui Z, Jing L, Guojun Z, Hong L. Epidemiology and risk factors of neurosurgical bacterial meningitis/encephalitis induced by carbapenem resistant Enterobacteriaceae. J Infect Chemother. 2020;26(1):101-6.

28. Petti CA, Woods CW, Reller LB. Streptococcus pneumoniae antigen test using positive blood culture bottles as an alternative method to diagnose pneumococcal bacteremia. J Clin Microbiol. 2005;43(5):2510-2.

29. Piette $A$, Verschraegen $G$. Role of coagulase-negative staphylococci in human disease. Vet Microbiol. 2009;134(1-2):45-54.

30. Assegu Fenta D, Lemma K, Tadele H, Tadesse BT, Derese B. Antimicrobial sensitivity profile and bacterial isolates among suspected pyogenic meningitis patients attending at Hawassa University Hospital: cross-sectional study. BMC Microbiol. 2020;20(1):125

31. Le J, Bookstaver PB, Rudisill CN, Hashem MG, lqbal R, James CL, et al. Treatment of meningitis caused by vancomycin-resistant Enterococcus faecium: high-dose and combination daptomycin therapy. Ann Pharmacother. 2010:44(12):2001-6.

32. Jaspan HB, Brothers AW, Campbell AJ, McGuire JK, Browd SR, Manley TJ, et al. Multidrug-resistant Enterococcus faecium meningitis in a toddler: characterization of the organism and successful treatment with intraventricular daptomycin and intravenous tigecycline. Pediatr Infect Dis J. 2010;29(4):379-81.

33. Inan D, Gunseren F, Colak D, Saba R, Kazan S, Mamikoglu L. First confirmed case of vancomycin-resistant Enterococcus faecium meningitis in Turkey: case report and literature review. J Chemother. 2004;16(6):608-11.

34. Sharma R, Goda R, Borkar SA, Katiyar V, Agarwal S, Kumar A, et al. Outcome following postneurosurgical Acinetobacter meningitis: an institutional experience of 72 cases. Neurosurg Focus. 2019;47(2).

35. Li Q, Zhu J, Kang J, Song Y, Yin D, Guo Q, et al. Emergence of NDM-5-producing carbapenem-resistant Klebsiella pneumoniae and SIM-producing hypervirulent Klebsiella pneumoniae Isolated from aseptic body fluid in a large tertiary hospital, 2017-2018: genetic traits of blaNDM-like and blaSIMlike genes as determined by NGS. Infect Drug Resist. 2020;13:3075-89.

36. Li L, Zhu C, Huang H. Clinical epidemiology and outcomes of biliary tract infections caused by Klebsiella pneumoniae. Ann Transl Med. 2019;7(14):304.

37. Chang JB, Chen $Y$, Wang $H, M a X$, Zhang $X$, Wu H, et al. Combined strategy for post-operative patients with central nervous system infections caused by extensively drug-resistant/pan-drug-resistant Acinetobacter baumannii: a retrospective study. Surg Infect (Larchmt). 2020;21(10):853-8.

38. Velkov T, Dai C, Ciccotosto GD, Cappai R, Hoyer D, Li J. Polymyxins for CNS infections: pharmacology and neurotoxicity. Pharmacol Ther. 2018;181:85-90

39. Michalopoulos A, Karatza D. Multidrug-resistant Gram-negative infections: the use of colistin. Expert Rev Anti Infect Ther. 2010;8(9):1009-17.

40. Tsuji BT, Pogue JM, Zavascki AP, Paul M, Daikos GL, Forrest A, et al. International consensus guidelines for the optimal use of the polymyxins: endorsed by the American College of Clinical Pharmacy (ACCP), European Society of Clinical Microbiology and Infectious Diseases (ESCMID), Infectious Diseases Society of America (IDSA), International Society for Anti-infective Pharmacology (ISAP), Society of Critical Care Medicine (SCCM), and Society of Infectious Diseases Pharmacists (SIDP). Pharmacotherapy. 2019:39(1):10-39.

41. Li LM, Zheng WJ, Shi SW. Spinal arachnoiditis followed by intrathecal tigecycline therapy for central nervous system infection by extremely drug-resistant Acinetobacter baumannii. J Int Med Res. 2020;48(7):300060520920405.

42. Mao DH, Miao JK, Zou X, Chen N, Yu LC, Lai X, et al. Risk factors in predicting prognosis of neonatal bacterial meningitis-a systematic review. Front Neurol. 2018;9:929.

43. Shi YJ, Zheng GH, Qian LY, Qsman RA, Li GG, Zhang GJ. Longitudinal analysis of risk factors for clinical outcomes of enterobacteriaceae meningitis/ encephalitis in post-neurosurgical patients: a comparative cohort study during 2014-2019. Infect Drug Resist. 2020;13:2161-70.

44. Wada M, Takeshima T, Nakamura Y, Nagasaka S, Kamesaki T, Kajii E, et al. Association between smoking and the peripheral vestibular disorder: a retrospective cohort study. Sci Rep. 2017;7(1):16889.

\section{Publisher's Note}

Springer Nature remains neutral with regard to jurisdictional claims in published maps and institutional affiliations. 